\title{
ON CURVES CONTAINED IN CONVEX SUBSETS OF THE PLANE
}

\author{
DON COPPERSMITH, GYOZO NAGY, AND SASHA RAVSKY
}

\begin{abstract}
If $K^{\prime} \subset K$ are convex bodies of the plane then the perimeter of $K^{\prime}$ is not greater than the perimeter of $K$. We obtain the following generalization of this fact. Let $K$ be a convex compact body of the plane with the perimeter $p$ and the diameter $d$ and $r>1$ be an integer. Let $s$ be the smallest number such that for any curve of length greater than $s$ contained in $K$ there is a straight line intersecting the curve at least in $r+1$ different points. Then $s=r p / 2$ if $r$ is even and $s=(r-1) p / 2+d$ if $r$ is odd.
\end{abstract}

Let $K$ be a compact subset of the plane. Recall that the set $K$ is called convex if for each pair of points $x, y \in K$ the segment $[x, y]$ connecting these points belongs to $K$. The set $K$ is called strictly convex if for each pair of points $x, y \in K$ the interval $(x, y)=[x, y] \backslash\{x, y\}$ belongs to the interior int $K$ of the set $K$. Equivalently, the set $K$ is strictly convex if its boundary $d K$ contains no segments of nonzero length.

Let $I=[0 ; 1]$ be the unit segment. A curve is a continuous map $\varphi: I \rightarrow \mathbb{R}^{2}$. Let $0=a_{0}<\cdots<a_{n}=1$ be a sequence. Let $[\varphi]$ be the broken line with the sequence of vertices $\varphi\left(a_{0}\right), \ldots, \varphi\left(a_{n}\right)$ that is the union of the segments $\bigcup_{i=0}^{n-1}\left[\varphi_{i} ; \varphi_{i+1}\right]$. By $l\left(a_{0}, \ldots, a_{n}\right)$ we denote the length of the broken line $[\varphi]$. We say that the curve $\varphi$ has length $l(\varphi)$ if for each $\varepsilon>0$ there is $\delta>0$ such that $\left|l(\varphi)-l\left(a_{0}, \ldots, a_{n}\right)\right|<\varepsilon$ for any sequence $0=a_{0}<\cdots<a_{n}=1$ with $a_{i+1}-a_{i}<\delta$ for each $0 \leq i \leq n-1$.

The convex subset $K$ of the plane is called a convex body if int $K \neq \emptyset$. If $K$ is a convex body then the boundary $d K$ of $K$ is an image of a curve and there exists the length $l(d K)$ which is equal to the upper bound of the perimeters of convex polygons 11 inscribed in $K$ [Ale, p. 373],[YB, p.28]. The length of the boundary we shall call the perimeter of the body $K$ and shall denote as $p(K)$.

It is well known that $p\left(K^{\prime}\right) \leq p(K)$ for every pair of convex bodies $K$ and $K^{\prime}$ with $K^{\prime} \subset K$ ([Ale, p. 373],[YB, p.28]). This means that if the image of a curve $\varphi: I \rightarrow K$ is a boundary of a convex body then $l(\varphi) \leq p(K)$. A slightly weaker claim is: if the image of a curve $\varphi: I \rightarrow K$ is a boundary of a strictly convex body then $l(\varphi) \leq p(K)$. The boundary $d K^{\prime}$ of the strictly convex body $K^{\prime}$ has the property that every line intersects $d K^{\prime}$ in at most two points. We are going to generalize the claim showing that $l(\varphi) \leq p(K)$ for every curve $\varphi: I \rightarrow K$ with every line intersecting the image of $\varphi$ at most in two points. This result is closely related with the following proposition.

Proposition 1. Let $S^{1}$ be a circle and $\varphi: S^{1} \rightarrow \mathbb{R}^{2}$ be a homeomorphic embedding. Then the following conditions are equivalent:

(1) $\varphi\left(S^{1}\right)$ is a boundary of a strictly convex body.

(2) Every line intersects the image of $\varphi$ at most in two points.

(3) Every line intersects the image of $\varphi$ at most in three points.

1991 Mathematics Subject Classification. 52A10, 52A38.

Key words and phrases. convex body, strictly convex body.

${ }^{1}$ We can consider a convex polygon as the convex hull of a broken line 
Proof. The implication $(1) \Rightarrow(2)$ follows from the definition of the strictly convex body and the implication $(2) \Rightarrow(3)$ is obvious. Now we show the implication $(3) \Rightarrow(1)$. Put $K=\operatorname{conv} \varphi\left(S^{1}\right)$. Since $\varphi$ is a homeomorphism then $K$ is not a segment and hence $K$ is a convex compact body. Let $x \in S^{1}$ be an arbitrary point. Let $C \subset \mathbb{R}^{2}$ be the circle of radius 1 with the center at the point $\varphi(x)$ and $p: \mathbb{R}^{2} \backslash\{\varphi(x)\} \rightarrow C$ be the radial projection. Since the map $p$ is continuous the set $p \varphi\left(S^{1} \backslash\{x\}\right)$ is connected and therefore an arc. Suppose that the length of $p \varphi\left(S^{1}\right)$ is greater than $\pi$. Then there exist points $x_{1}, x_{2}, x_{3} \in S^{1} \backslash\{x\}$ such that the point $\varphi(x)$ lies in the interior of the triangle with the vertices $p \varphi\left(x_{1}\right), p \varphi\left(x_{2}\right)$ and $p \varphi\left(x_{3}\right)$. Then the point $\varphi(x)$ lies in the interior of the triangle with the vertices $\varphi\left(x_{1}\right), \varphi\left(x_{2}\right)$ and $\varphi\left(x_{3}\right)$. Fix an orientation on the circle $S^{1}$. After a re-enumerating we may suppose that the points lie on the circle $S^{1}$ in the order $x, x_{1}, x_{2}$, $x_{3}$. Let $l \subset \mathbb{R}^{2}$ be a line separating the points $\varphi(x)$ and $\varphi\left(x_{2}\right)$ from the points $\varphi\left(x_{1}\right)$ and $\varphi\left(x_{3}\right)$. Since the images under the map $\varphi$ of the oriented $\operatorname{arcs}\left(x, x_{1}\right),\left(x_{1}, x_{2}\right),\left(x_{2}, x_{3}\right)$ and $\left(x_{3}, x\right)$ are linearly connected then each of the images contain a point from the line $l$. Obtained contradiction shows that the length of the $\operatorname{arc} p \varphi\left(S^{1} \backslash\{x\}\right)$ is not greater than $\pi$.

Therefore there is a line $l$ going through the point $\varphi(x)$ such that one of the open half planes created by $l$ contains no points of the set $p \varphi\left(S^{1} \backslash\{x\}\right)$ and hence no points of the set $\varphi\left(S^{1}\right)$. This implies that the point $\varphi(x)$ is a boundary point of the set $K$.

Hence $\varphi\left(S^{1}\right) \subset d K$. Since $d K$ is homeomorphic to a circle [Ale, p. 372] and $\varphi\left(S^{1}\right) \subset d K$ is homeomorphic to a circle the $\varphi\left(S^{1}\right)$ must coincide with $d K$. The set $K$ is strictly convex since its boundary $\varphi\left(S^{1}\right)$ contains no segments of nonzero length.

The main result of the paper is the following

Theorem 1. Let $K \subset \mathbb{R}^{2}$ be a convex compact body with perimeter $p$, diameter $d$ and let $r>1$ be an integer. Let $s$ be the smallest number such that for any curve $\varphi \subset K$ of length greater than $s$ there is a line intersecting the curve $\varphi$ at least in $r+1$ different points. Then

$$
s=\left\{\begin{array}{l}
r p / 2, \text { if } r \text { is even } \\
(r-1) p / 2+d, \text { if } r \text { is odd }
\end{array}\right.
$$

Proof. Fix the compact body $K$ and the number $r$. Let $p$ be the perimeter of $K$ and $d$ be the diameter of $K$.

The upper bound. Put $s=r p / 2$ if $r$ is even and $s=(r-1) p / 2+d$ if $r$ is odd. Let $\varphi: I \rightarrow K$ be a curve of length greater than $s$. By the definition of the length we can choose numbers $0=a_{0}<\ldots<a_{n}=1$ such that the length of the broken line with the sequence of vertices $\varphi\left(a_{0}\right), \ldots, \varphi\left(a_{n}\right)$ is greater than $s$. Let $l_{1}, \ldots, l_{n}$ be the lengths of the segments of the broken line and $\alpha_{1}, \ldots, \alpha_{n}$ be the angles with these segments and the $O x$ axis. For an angle $\alpha \in[0 ; 2 \pi]$, by $l(\alpha)$ we denote the sum of the lengths of the projections of the segments $l_{i}$ onto the line $L_{\alpha}=\{(x, y):(x, y)=t(\cos \alpha, \sin \alpha)\}$. Thus $l(\alpha)=\sum l_{i}\left|\cos \left(\alpha-\alpha_{i}\right)\right|$. By $k(\alpha)$ we denote the length of the projection of $K$ onto the line $L_{\alpha}$. By Cauchy formula [Had, 6.1.5]

$$
\int_{0}^{2 \pi} k(\alpha) d \alpha=2 p .
$$


At first suppose that $r$ is even. To the rest of the proof it suffice to show that there is an angle $\alpha^{\prime}$ such that $l\left(\alpha^{\prime}\right)>r k\left(\alpha^{\prime}\right)$. Suppose the contrary. Then

$$
\begin{gathered}
2 r p=r \int_{0}^{2 \pi} k(\alpha) d \alpha \geq \int_{0}^{2 \pi} l(\alpha) d \alpha=\int_{0}^{2 \pi} \sum l_{i}\left|\cos \left(\alpha-\alpha_{i}\right)\right| d \alpha= \\
\sum \int_{0}^{2 \pi} l_{i}\left|\cos \left(\alpha-\alpha_{i}\right)\right| d \alpha=\sum \int_{0}^{2 \pi} l_{i}|\cos (\beta)| d \beta=\sum 4 l_{i}>4 s=2 r p,
\end{gathered}
$$

the contradiction.

Now suppose that $r$ is odd. Add to the broken line the segment connecting its ends. Let the length of the segment be $l_{0}$ and $\alpha_{0}$ be the angle with the segment and the $O x$ axis. To the rest of the proof it suffice to show that there is an angle $\alpha^{\prime}$ such that $l\left(\alpha^{\prime}\right)>r l_{0}\left|\cos \left(\alpha^{\prime}-\alpha_{0}\right)\right|+(r-1)\left(k\left(\alpha^{\prime}\right)-l_{0}\left|\cos \left(\alpha^{\prime}-\alpha_{0}\right)\right|\right)=(r-1) k\left(\alpha^{\prime}\right)+l_{0}\left|\cos \left(\alpha^{\prime}-\alpha_{0}\right)\right|$. That is because each line not intersecting $l_{0}$ and the vertices of the broken line intersects the broken line an even number of times.

Suppose the contrary. Then

$$
\begin{gathered}
2(r-1) p+4 l_{0}=\int_{0}^{2 \pi}(r-1) k(\alpha)+l_{0}\left|\cos \left(\alpha-\alpha_{0}\right)\right| d \alpha \geq \int_{0}^{2 \pi} l(\alpha) d \alpha=\int_{0}^{2 \pi} \sum l_{i}\left|\cos \left(\alpha-\alpha_{i}\right)\right| d \alpha= \\
\sum \int_{0}^{2 \pi} l_{i}\left|\cos \left(\alpha-\alpha_{i}\right)\right| d \alpha=\sum \int_{0}^{2 \pi} l_{i}|\cos (\beta)| d \beta=\sum 4 l_{i}>4 s=2(r-1) p+4 d,
\end{gathered}
$$

which yields the contradiction since $l_{0} \leq d$.

The lower bound. The idea of the proof is the following. Put $n=\lfloor r / 2\rfloor$. If $r$ is even, let the curve go $n$ times around the perimeter, curving slightly to avoid any straight lines but remaining convex. If $r$ is odd, let the curve go almost $n$ times around the perimeter, then down the diameter, again always curving slightly. The reason we go "almost" $n$ times around the perimeter is to ensure that any straight line intersecting this "curved diameter" twice, will intersect the perimeter-curve at most $r-2$ times rather than $r-1$. The picture illustrates the construction when $K$ is a square and $r=5$.

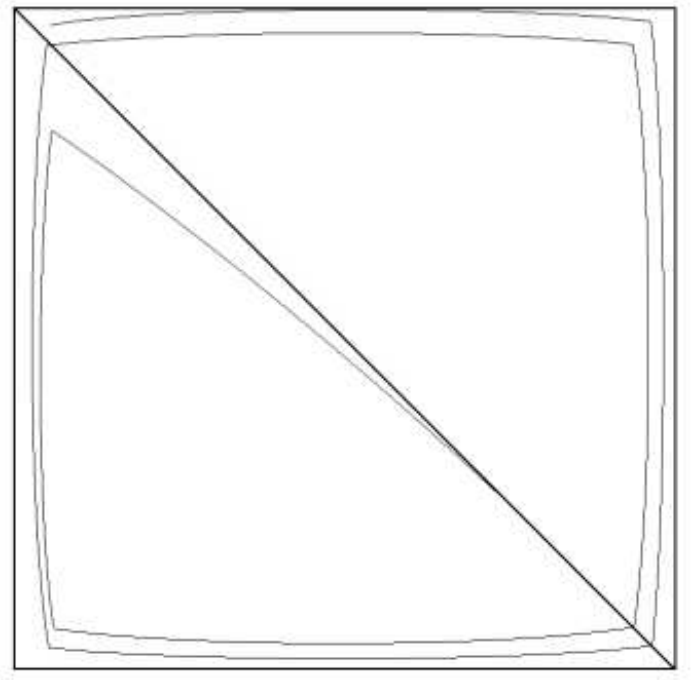

Now more precisely. Let $\varepsilon>0$. At first we suppose that $r$ is even. There is a curve $\varphi_{1}: I \rightarrow K$ such that $\varphi_{1}(0)=\varphi_{1}(1),\left|l\left(\varphi_{1}\right)-p\right|<\varepsilon$ and $\varphi_{1}(I)$ is the boundary of a 
strictly convex body. Choose a number $\delta_{1}>0$ such that $l\left(\varphi_{1}\left(\left[1-\delta_{1} ; 1\right]\right)\right)<\varepsilon$. Similarly, there is a curve $\varphi_{2}: I \rightarrow K$ such that $\varphi_{2}(0)=\varphi_{2}(1),\left|l\left(\varphi_{1}\right)-l\left(\varphi_{2}\right)\right|<\varepsilon, \varphi_{2}(I)$ is the boundary of a strictly convex body, $\varphi_{2}(0)=\varphi_{1}(1-\delta)$ and $\varphi_{2}((0 ; 1)) \subset \operatorname{int} \operatorname{conv} \varphi_{1}(I)$. Choose a number $\delta_{2}>0$ such that $l\left(\varphi_{2}\left(\left[1-\delta_{2} ; 1\right]\right)\right)<\varepsilon$. Similarly to the previous we can construct the curve $\varphi_{3}: I \rightarrow K$ and so on. From the curves $\varphi_{1}, \ldots, \varphi_{n}$ it is easily to construct the curve $\varphi$ intersecting every straight line in at most $r$ points.

Now suppose that $r$ is odd. To construct the curve $\varphi$ we shall proceed similarly to the case of even $n$. By induction we can construct the curves $\varphi_{1}, \ldots, \varphi_{n}$ such that

(i) $\left|\operatorname{diam}\left(\varphi_{1}(I)\right)-\operatorname{diam}(K)\right|<\varepsilon$,

(ii) $\left|\operatorname{diam}\left(\varphi_{i}(I)\right)-\operatorname{diam}\left(\varphi_{i-1}(I)\right)\right|<\varepsilon$ for each $i$,

(iii) there is a number $\alpha_{i}$ such that $\left|\operatorname{diam}\left(\varphi_{i}(I)\right)-\right| \varphi_{i}(0)-\varphi_{i}\left(\alpha_{i}\right)||<\varepsilon$ for each $i$.

Now choose a number $\delta_{n}>0$ such that $\left|\operatorname{diam}\left(\varphi_{n}(I)\right)-\right| \varphi_{i}\left(1-\delta_{n}\right)-\varphi_{i}\left(\alpha_{i}\right)||<\varepsilon$ and $l\left(\varphi_{n}\left(\left[1-\delta_{n} ; 1\right]\right)\right)<\varepsilon$. There exists a curve $\varphi_{n+1}: I \rightarrow K$ such that

(i) $\varphi_{n+1}(0)=\varphi_{n}\left(1-\delta_{n}\right)$ and $\varphi_{n+1}(1)=\varphi_{n}\left(\alpha_{n}\right)$,

(ii) $\varphi_{n+1}((0 ; 1))$ lies in the interior of the triangle with the vertices $\varphi_{n}(0), \varphi_{n}\left(\alpha_{n}\right)$ and $\varphi_{n}\left(1-\delta_{n}\right)$,

(iii) $\varphi_{n+1}(I) \cup\left[\varphi_{n}\left(1-\delta_{n}\right) ; \varphi_{n}\left(\alpha_{n}\right)\right]$ is the boundary of a convex body,

(iv) no three points of the set $\varphi_{n+1}(I)$ lie on a straight line.

From the curves $\varphi_{1}, \ldots, \varphi_{n+1}$ it is easy to construct the curve $\varphi$ intersecting every straight line in at most $r$ points.

\section{REFERENCES}

[Ale] A.D. Aleksandrov. Inner geometry of convex surfaces, OGIZ, 1948. (in Russian)

[Gash] S.B. Gashkov. Problem M932 // Kvant - 1985. - V. 11. - P.36-37. (in Russian)

[Had] H. Hadwiger. Vorlesungen über inhalt, oberfläche und isoperimetrie, Springer-Verlag, 1957. (in Russian, M.:Nauka, 1966)

[YB] I.M. Yaglom, V.G. Boltyanskiy. Convex figures, GITTL, 1951. (in Russian)

E-mail address: dcopper@us.ibm.com;gyozo.nagy@raiffeisen.hu; oravsky@mail.ru

Department of Mathematical Sciences, iBm Thomas J Watson Research Center, Yorktown Heights Ny 10598, USA; Technical Center, Raiffeisen Hungary, Budapest Kesmark 11-13, 1158, Hungary; Department of Mathematics, Ivan Franko Lviv National UniverSITY, UNIVERSYTETSKa 1, LVIV, 79000, UKRAINE 